\title{
La estrategia curricular del inglés en las asignaturas de Física en la carrera de Técnico superior de Biofísica
} Médica

The curricular strategy of the English language in the Physics subjects for Medical Biophysics Senior Technicians

Alexander Torres Hernández. ${ }^{1}$, Magdelin Rojas Rosales. ${ }^{2}$, Yamila Álvarez Góngora. ${ }^{3}$ \& Adina Suárez Ceijas. ${ }^{4}$

\section{DOI: https://doi.org/10.33262/anatomiadigital.v3i3.1281}

Abstract.

The ways to implement the English language as a curricular strategy are not established in the subjects: Applied Physics and Physics of Radiations syllabuses for the major in Medical Biophysics Senior Technicians. Accordingly, the objective is to provide some methodological guidelines in order to implement the curricular strategy of the English language in the subjects: Applied

\section{Resumen.}

En los programas de las asignaturas Física Aplicada y Física de las Radiaciones de la carrera de Técnico Superior en Biofísica Médica no se establecen vías para implementar la estrategia curricular de idioma inglés. Por esta razón, el objetivo es presentar orientaciones metodológicas para implementar la estrategia curricular del idioma inglés en las asignaturas Física Aplicada y Física de las Radiaciones. Para

\footnotetext{
${ }^{1}$ Universidad de Ciencias Médicas de Matanzas. Facultad de Ciencias Médicas Juan Guiteras Gener.

Matanzas, Cuba. alexanderth.mtz@infomed.sld.cu@ ID https://orcid.org/0000-0002-9235-410X

${ }^{2}$ Universidad de Ciencias Médicas de Matanzas. Facultad de Ciencias Médicas Juan Guiteras. Matanzas, Cuba.magdelinrojas@gmail.com iD https://orcid.org/0000-0003-3908-1197

${ }^{3}$ Universidad de Ciencias Médicas de Matanzas. Facultad de Ciencias Médicas Matanzas Juan Guiteras.

Matanzas, Cuba. yalavarezgongora@gmail.com iD https://orcid.org/0000-0002-1753-4151

${ }^{4}$ Universidad de Ciencias Médicas de Matanzas. Facultad de Ciencias Médicas Matanzas Juan Guiteras.

Matanzas, Cuba. adinasuarez1968@gmail.com iD https://orcid.org/0000-0001-6830-9037
} 
Physics and Physics of Radiations. For the development of this work, the analysissynthesis and the inductive-deductive methods were used with a systemic approach. With the elaboration of these methodological guidelines, the syllabuses are perfected. That way, they favor the implementation of the curricular strategy of the English language in the subjects already mentioned.

Keywords: Curricular strategy; Medical Higher Education; Physics in the health area; Medical Biophysics; Teaching of Physics. ello se utilizaron métodos de análisis y síntesis, inducción y deducción con un enfoque de sistema. Se perfeccionan los programas, a partir de la elaboración de orientaciones metodológicas. De esa forma, ellas favorecen la implementación de la estrategia curricular del idioma inglés en las asignaturas anteriormente referidas.

Palabras claves: Estrategia curricular; Educación Médica Superior; Física en Salud; Biofísica Médica; Enseñanza de la Física.

\section{Introducción.}

En el curso 2019-2020 inicia en la Universidad de Ciencias Médicas de Matanzas, la formación de Técnico Superior de Biofísica Médica. Según se establece en el plan de estudio, los graduados de esta carrera poseen "una sólida preparación general integral y profesional básica en la explotación de los equipos, que les permite enfrentar los problemas de su profesión, analizar la solución y ejecutar las actividades con independencia y creatividad” (Ministerio de Educación Superior, 2018, pág. 4).

Para lograr esos propósitos las asignaturas Física Aplicada y Física de las Radiaciones que se imparten en el primer y segundo semestre respectivamente, están diseñadas para que los estudiantes se apropien de los conocimientos físicos necesarios para comprender fenómenos fisiológicos del cuerpo humano y del área de la Radiología para el diagnóstico y tratamiento con radiaciones y el empleo de la medicina nuclear.

Otro de los aspectos que se conciben desde el diseño curricular es que los estudiantes logren comunicarse con un nivel post-intermedio en las cuatro habilidades fundamentales del idioma inglés (Rodríguez Sánchez, 2018). Al respecto en las indicaciones para instrumentar las estrategias curriculares que se presentan en el modelo del profesional se plantea que: "se realizará un trabajo integral entre la asignatura inglés y las demás asignaturas, todo lo cual contribuirá a que los estudiantes sean capaces de utilizar adecuadamente este idioma por la importancia que tiene en el desarrollo de su labor profesional en el ámbito nacional como internacional" (Ministerio de Salud Pública. Universidad de Ciencias Médicas de la Habana, 2018, pág. 6).

En las orientaciones metodológicas de los programas de las asignaturas Física Aplicada y Física de las Radiaciones no se hace referencia a cómo abordar durante el proceso de enseñanza-aprendizaje la estrategia curricular del idioma inglés. Queda a la espontaneidad de los profesores de Física. 
Como parte del perfeccionamiento de los programas de las asignaturas de Física Aplicada y Física de las Radiaciones, se consideró realizar un trabajo metodológico entre profesores de inglés y de Física con el objetivo de presentar orientaciones metodológicas para implementar la estrategia curricular del idioma inglés en estas asignaturas. A partir de las indicaciones establecidas desde el diseño curricular en los documentos normativos y legales de la carrera, así como de las limitaciones metodológicas existentes en los referidos programas.

\section{Metodología.}

Se realizó un trabajo metodológico conjunto entre profesores de inglés y de Física para elaborar las orientaciones metodológicas. Para ello se utilizaron métodos de análisis y síntesis, inducción y deducción con un enfoque de sistema. Participaron los autores del presente texto, un profesor de Física que se desempeña como coordinador de la carrera y tres profesoras de inglés.

Se realizaron tres reuniones de trabajo entre los meses de septiembre y noviembre de 2019 en el que se intercambiaron documentos normativos de la carrera, entre estos, los programas de las asignaturas de inglés, Física Aplicada y Física de las Radiaciones. Además, se analizó el modelo del profesional en los que se consideraron los objetivos generales de la carrera, el objeto de la profesión, el modo de actuación de estos profesionales y el campo de acción en el que pueden desempeñarse. Se analizaron los objetivos de las asignaturas, el sistema de conocimientos y las habilidades a desarrollar en cada una.

Se realizaron diversas propuestas de cómo introducir en los diferentes temas de las asignaturas de Física el idioma inglés y se llegó a consensos en cómo realizarlo. Se presentó en el colectivo de carrera en enero de 2020 y fueron aprobadas las orientaciones metodológicas.

\section{Resultados.}

Para abordar el idioma inglés en las asignaturas Física Aplicada y Física de las Radiaciones se debe valorar su utilización durante las clases en aspectos como la introducción de magnitudes, unidades y ecuaciones en su forma inglesa. Se puede presentar de manera progresiva pequeños textos en inglés sobre algún aspecto específico del contenido que se imparte en cualquiera de las formas organizativas del proceso de enseñanza-aprendizaje.

En las clases prácticas se puede utilizar al menos uno de los ejercicios o problemas en inglés. Para ello el profesor debe prepararse en cuanto a la traducción correcta y explicar de manera adecuada el significado del texto. En ese proceso debe permitirles un tiempo a los estudiantes para que analicen el ejercicio propuesto y que expresen que comprendieron. Se deben realizar todas las preguntas necesarias para favorecer el 
intercambio, el diálogo y la interpretación desde el punto de vista físico de la situación propuesta.

Es necesario que en todas las clases se propongan para el estudio independiente y realización de tareas extraclases, bibliografía en inglés en las que se aborden los conocimientos que se impartieron y algunas que aborden aplicaciones de la física. El profesor debe realizar orientaciones específicas sobre los aspectos más importantes de los textos que proponen y que utilizarán los estudiantes. Además, se le orientarán el empleo de diccionarios bilingües de español-inglés y monolingües en inglés.

En la media en que los estudiantes desarrollen la habilidad de lectura en inglés, se sugiere aplicar problemas o preguntas teóricas en comprobaciones escritas y en algunas de las preguntas en los exámenes parciales y finales. El propósito es familiarizar al inicio hasta lograr desarrollar las habilidades de lectura e interpretación de textos en inglés sobre contenidos físicos que tienen aplicación en el objeto de la profesión.

Se puede contribuir a la habilidad de la escritura cuando se les oriente a los estudiantes escribir las respuestas de los ejercicios, problemas y preguntas teóricas que se aplican durante las clases de conferencias, prácticas y de estudio independiente. Esto implica una superación constante de los profesores de física y un análisis previo de toda la bibliografía complementaria que se orienta y de las respuestas correctas no solo desde la física, también desde el idioma inglés.

A modo de ejemplo se presenta algunas de las actividades que se pueden realizar y que se corresponden con las orientaciones metodológicas que se elaboraron.

La asignatura Física Aplicada está compuesta por los diferentes temas, lo cuales se presentan en la siguiente tabla.

Tabla 1. Fondo de tiempo por temas y formas de organización de la enseñanza

\begin{tabular}{llccccc}
\hline \multicolumn{1}{c}{ Temática } & CONF & SEM & CT-P & EVAL & $\begin{array}{c}\text { Total de } \\
\text { Horas }\end{array}$ \\
\hline 1. Magnitudes físicas & 2 & 1 & 2 & & 5 \\
2. Fuerza, Cantidad de Movimiento y Energía & 3 & & 1 & & 4 \\
3. Oscilaciones y ondas & 4 & 1 & 1 & & 6 \\
& Trabajo de Control Parcial & & & & 1 & 1 \\
4. Campo eléctrico y corriente eléctrica & 4 & 1 & 2 & & 6 \\
5. $\begin{array}{l}\text { Campo magnético e inducción } \\
\text { electromagnética }\end{array}$ & 3 & 1 & 2 & & 6 \\
6. Ondas electromagnéticas & 4 & 1 & & & 5 \\
& Examen Final & & & & 2 & 2 \\
\hline
\end{tabular}


Leyenda: CONF: Conferencias ｜ SEM: Seminarios |CT-P: Clases TeóricoPrácticas

Fuente: (Calderón Marín, 2018, pág. 4)

\section{Para la temática 1. Magnitudes físicas.}

\section{“Contenidos:}

Introducción. Magnitudes físicas. Cantidad y unidad. Unidades fundamentales y derivadas. Análisis dimensional. Constantes físicas. Sistemas de unidades. Cifras significativas y redondeo. Medición experimental. Mediciones directas e indirectas. Fuentes de errores en las mediciones experimentales. Error aleatorio y error sistemático. Media aritmética, desviación estándar y coeficiente de variación. Error relativo. Test de chi-cuadrado. Precisión y exactitud. Calibraciones y patrones de medida." (Calderón Marín, 2018, pág. 5)

En esta temática cuando se trate las cantidades y unidades, además de realizar la explicación en español, se puede introducir el nombre de las unidades básicas de longitud, tiempo y masa en inglés, estas serían, Length, Time y Mass. De esta forma sin que se convierta en una clase de inglés se debe incorporar el vocabulario de los aspectos principales del sistema de conocimientos y que utilizarán en la lectura y escritura de textos en idioma inglés.

\section{Para la temática 6 Ondas Electromagnéticas}

\section{“Contenidos:}

Ondas electromagnéticas. Naturaleza de las ondas electromagnéticas. Longitud de onda, frecuencia y energía de una onda electromagnética. Espectro electromagnético. Propagación de las ondas electromagnéticas. Radiación. Radiaciones no ionizantes. Carácter dual de las ondas electromagnéticas. Luz visible, luz ultravioleta y radiación infrarroja. Láser. Resonancia Magnética. Principios físicos de la formación de imágenes de resonancia magnética. Interacción de las radiaciones electromagnéticas no ionizantes con la materia y los sistemas biológicos. Aplicaciones biomédicas de las radiaciones electromagnéticas no ionizantes.” (Calderón Marín, 2018, pág. 7)

Otra de las vías que se plantean es la utilización de ejercicios y problemas escritos en inglés. Para ello el profesor debe tomar en cuenta que el texto que propone este acorde al nivel lingüístico en idioma inglés de los estudiantes y que las fuentes de estos deben ser autores de habla inglesa (Rodríguez Santana, Valdivia Sardiñas, \& Zayas Tamayo, 2016).

Uno de los problemas que pueden ser utilizados para desarrollar habilidades en el cálculo del potencial eléctrico, es el siguiente: 
"A solid conducting sphere of radius has a total charge Find the electric potential everywhere, both outside and inside the sphere.” (Young, Freedman, \& Ford, 2012, pág. 768)

El profesor debe permitirles que analicen correctamente el texto y realizar preguntas sobre la interpretación del mismo y la comprensión de las condiciones que se presentan en el ejercicio. Para ello realizará preguntas que se relacionen con la descripción de la situación física para asegurar que los estudiantes mediante el intercambio dialógico logren establecer el procedimiento a seguir para solucionarlo e interpretar los resultados. La respuesta del ejercicio se escribirá en inglés y se llegará a un consenso entre los estudiantes y el profesor en cual fue mejor escrita y se les evaluará la actividad realizada. Es necesario reflexionar junto con los estudiantes en cuanto a la utilidad del aprendizaje del inglés como parte de la formación profesional y que algunas de las habilidades que deben desarrollar es la de escritura y lectura en este idioma.

Tabla 2. La asignatura Física de las Radiaciones está distribuida por los siguientes temas: Fondo de tiempo por temas y formas de organización de la enseñanza

\begin{tabular}{|c|c|c|c|c|c|c|}
\hline Temática & CONF & SEM & CT-P & LAB & EVAL & $\begin{array}{c}\text { Total de } \\
\text { Horas }\end{array}$ \\
\hline $\begin{array}{l}\text { 1. Conceptos y magnitudes de la } \\
\text { Física de Radiaciones }\end{array}$ & 1 & & & & & 1 \\
\hline 2. Estructura atómica y nuclear & 2 & & & & & 2 \\
\hline 3. Desintegración radiactiva & 6 & & 3 & 1 & & 10 \\
\hline $\begin{array}{l}\text { Trabajo de Control Parcial } \\
\text { 4. Interacción de la radiaciones } \\
\text { ionizantes con la materia }\end{array}$ & 4 & 1 & 2 & 1 & 1 & 1 \\
\hline 5. Rayos X & 3 & 2 & & & & 5 \\
\hline 6. Producción de radionucleidos & 3 & 3 & 1 & & & 7 \\
\hline Examen Final & & & & & 2 & 2 \\
\hline TOTAL & 19 & 6 & 6 & 2 & 3 & 36 \\
\hline
\end{tabular}

Leyenda: CONF: Conferencias ｜ SEM: Seminarios | CT-P: Clases TeóricoPrácticas |LAB: Laboratorios EVAL: Evaluaciones

Fuente: (Calderón Marín C. F., 2018, pág. 3)

En el Tema 4. Interacción de las radiaciones ionizantes con la materia, está presente en el sistema de conocimientos el efecto fotoeléctrico, para este puede utilizarse el siguiente ejercicio.

"While conducting a photoelectric-effect experiment with light of a certain frequency, you find that a reverse potential difference of $1.25 \mathrm{~V}$ is required to reduce the current to zero. Find (a) the máximum kinetic energy and (b) the maximum speed of the emitted." (Young, Freedman, \& Ford, 2012, pág. 1266) 
Se mantiene el tratamiento metodológico descrito en este material. Estos son ejercicios que ejemplifican como pueden utilizarse. Existen una gran cantidad de literatura de física general escrita por autores de lengua inglesa que son accesible a los profesores de física de la universidad de Ciencias Médicas y que pueden ser seleccionados por estos para emplearlos.

Siempre que sea posible se debe brindar la posibilidad a los estudiantes a responder de manera oral, algunas preguntas escritas u orales en inglés, del mismo modo para ejercicios y problemas que se hayan presentado en clase en ese idioma. Un aspecto importante es que esto no significa que las clases se desarrollen en inglés, pero tanto las conclusiones parciales y finales sobre aspectos específicos de los conocimientos físicos que se parten deben realizarse en español.

\section{Conclusiones:}

- Se perfeccionan los programas de Física Aplicada y Física de las Radiaciones de la carrera de Técnico Superior de Biofísica Médica en la Universidad de Ciencias Médicas de Matanzas, a partir de la elaboración de orientaciones metodológicas para la implementación de la estrategia curricular del idioma inglés.

- Las orientaciones metodológicas propuestas favorecen la implementación de la estrategia curricular del idioma inglés en las asignaturas Física Aplicada y Física de las Radiaciones.

\section{Referencias bibliográficas:}

Calderón Marín, C. (2018). Programa de la asignatura Física Aplicada. Ministerio de Salud Pública, La Habana.

Calderón Marín, C. F. (2018). Programa de Física de las Radiaciones. Universidad de Ciencias Médicas de la Habana, La Habana.

Ministerio de Educación Superior. (2018). Plan de estudio Educación Superior de Ciclo Corto de Biofísica Médica. La Habana.

Ministerio de Salud Pública. Universidad de Ciencias Médicas de la Habana. (2018). Modelo de formación profesional del nivel de educación superior de ciclo corto en Biofísica Médica. La Habana.

Rodríguez Sánchez, Y. (2018). Programa de Inglés para Técnico Superior (ciclo corto). Universidad de Ciencias Médicas de la Habana, La Habana.

Rodríguez Santana, M. M., Valdivia Sardiñas, M. d., \& Zayas Tamayo, A. (2016). El empleo del idioma inglés en el aprendizaje la FÍSICA GENERAL en la carrera de Licenciatura en Educación, MATEMÁTICA -FÍSICA. XI Taller Internacional "La Enseñanza de la Física y la Química” y IV Taller "La Enseñanza de las Ciencias Naturales". Matanzas.

Young, H., Freedman, R., \& Ford, A. (2012). Sears and Zemansky's university physics : with modern physics. San Francisco, USA: Addison-Wesley. 


\section{PARA CITAR EL ARTÍCULO INDEXADO.}

Torres Hernández, A., Rojas Rosales, M., Alvarez Góngora, Y., \& Suárez Ceijas, A. (2020). La estrategia curricular del inglés en las asignaturas de Física en la carrera de Técnico superior de Biofísica Médica. Anatomía Digital,3(3), 6-13. https://doi.org/10.33262/anatomiadigital.v3i3.1281

\section{Ciencia \\ LDigital}

El artículo que se publica es de exclusiva responsabilidad de los autores y no necesariamente reflejan el pensamiento de la Revista Anatomía Digital.

El artículo queda en propiedad de la revista y, por tanto, su publicación parcial y/o total en otro medio tiene que ser autorizado por el director de la Revista Anatomía Digital.
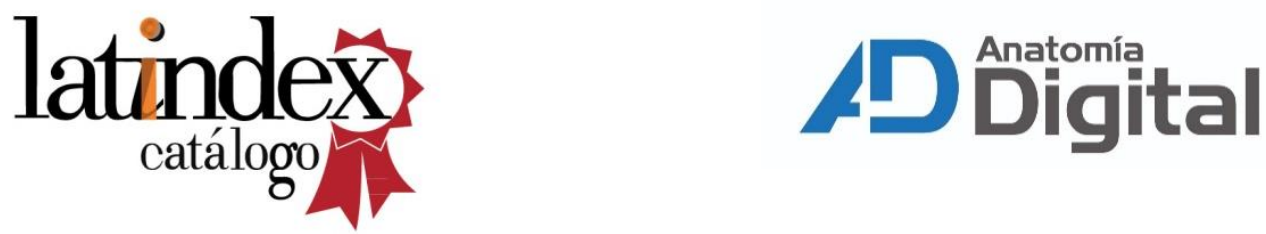